\title{
Rejection Degree by Fuzzy Significance Probability
}

\author{
Gyu-Tag Choi*†, Il-Soo Park**, Hyun-Woo Nam*** \\ and Jong-Choon Moon ${ }^{* * * *}$
}

(Received 18 October 2013, Revised 25 November 2013, Accepted 27 November 2013)

\begin{abstract}
We propose some properties for fuzzy hypothesis test by fuzzy significance probability. First, we define fuzzy number data and fuzzy significance probability for repeatedly observed data with alternated error term. By the agreement index, we compare fuzzy significance probability with significance level and drawing conclusions the degree of acceptance and rejection by agreement index.
\end{abstract}

Key Words : Fuzzy number data, Degree of acceptance and rejection, Fuzzy significance probability, Agreement index

\section{Introduction}

We propose some properties for fuzzy hypothesis test by fuzzy significance probability by agreement index. The negation of the assertion is taken to be the fuzzy null hypothesis $H_{f 0}$ and the assertion itself is taken to be the fuzzy alternative hypothesis $H_{f 1}$ by obtained data from the fuzzy samples[1].

Kang, Choi and Lee[2] defined fuzzy hypothesis membership function, also they found the agreement index by area for fuzzy hypothesis membership function and membership function of fuzzy critical region. Thus they obtained the results by the grade for judgement to acceptance or rejection for the

$* \dagger$ Gyu-Tag Choi(corresponding author) : Subdivision of Business Admniistration, KyungNam College of Information \& Technology.

E-mail : gtchoi@eagle.kit.ac.kr, Tel : 051-320-1376

**Il-soo Park : Dept. of Refrigeration \& Air-conditioning Engineering, TongMyong University.

***Hyun-Woo Nam : Dept. of Fire Safety Management, GyeongSan University

****Jong-Choon Moon : Dept. of Automative Engineering, GyeongSan University fuzzy hypothesis.

Thus, we introduction some properties of fuzzy hypothesis test using fuzzy significance probability by agreement index. First we define fuzzy number data for repeatedly observed data with alteration error term in random experiment. Comparing the fuzzy significance probability with significance level for the hypothesis, we draw conclusion for the degree of acceptance and rejection by agreement index.

For the fuzzy hypothesis, we consider

$$
H_{f 0}: \theta \simeq \psi \quad \text { or } \quad H_{f 0}: \theta<\psi, \theta \in \Theta
$$

where $" \simeq "$ is similarity and $"<"$ is less than or similarity. We construct a set

$$
\left\{\left(H_{f 0}(\psi), H_{f 1}(\psi)\right) \mid \psi \in \Theta\right\}
$$

with membership function $m_{H}(\psi)$ where $\Theta$ is parameter space.

For satisfying fuzzy statistical data, a fuzzy number $A$ in $\Re$ is said to be convex if for any real numbers $x, y, z \in \mathcal{R}$ with $x \leq y \leq z$, 


$$
m_{A}(y) \geqq m_{A}(x) \wedge m_{A}(z)
$$

with $\wedge$ standing for minimum.

Also, fuzzy statistical data $A$ is called normal if the following holds

$$
\bigvee_{x} m_{A}(x)=1
$$

An $\delta$-level set of a fuzzy data number $A$ is a set of $[A]^{\delta}$ and defined by

$$
[A]^{\delta}=\left\{x \mid m_{A}(x) \geqq \delta, 0<\delta \leqq 1\right\}
$$

An $\delta$-level set of fuzzy data number $A$ is a convex fuzzy set which is a closed and bounded interval denoted by $[A]^{\delta}=\left[A_{l}^{\delta}, A_{r}^{\delta}\right]$.

Let $A$ and $B$ be fuzzy numbers data in $\mathcal{R}$ and let $\odot$ be a binary operation defined in $\mathcal{R}$. Then the operation $\odot$ can be extended to the fuzzy numbers $A$ and $B$ by defining the relation of Zadeh's extension principle, as given $A, B \subset \Re, \forall x_{i}, x_{j}, x_{k} \in \Re$;

$$
\begin{aligned}
& m_{A_{\delta} \odot B_{\delta}}(z)=\bigvee_{x_{k}=x_{i} \odot x_{j}}\left(m_{A_{\delta}}\left(x_{i}\right) \wedge m_{B_{\delta}}\left(x_{j}\right)\right), \\
& i, j, k=1,2, \cdots
\end{aligned}
$$

A modelling the fuzziness of data were described the fuzziness of a fuzzy sample $x=\left(x_{1}, x_{2}, \cdots, x_{n}\right)$. As a precise fuzzy sample $x_{i}$ of $n$ precise realizations $x_{i} \in \Re$ may be regarded as vector in $\Re^{n}$.

\section{Acceptance or rejection degree}

Let a test statistics $T$ by fuzzy random sample from sample space $\Omega$. Let $\left\{P_{\theta}, \theta \in \Omega\right\}$ be a family of fuzzy probability distribution, where $\Theta$ is a parameter vector of $\Omega$.
Choose a membership function $m_{T}(x)$ whose value is likely to best reflect the plausibility of the fuzzy hypothesis being tested.

Let us consider membership function $m_{C}(x)$ of critical region $C$, which we will call the agreement index of $m_{T}(x)$ which regard to $m_{C}(x)([3],[5])$. Thus we have following agreement index.

Definition 2.1. Let a fuzzy membership function $m_{T}(x), x \in R$, and consider another membership function $m_{C}(x), x \in R$, then we have a index which call the agreement index as the ratio being defined in the following way;

$$
\operatorname{AGI}(T, C)=\frac{\operatorname{area}\left(m_{T}(x) \cap m_{C}(x)\right)}{\operatorname{area}\left(m_{C}(x)\right)} \in[0,1]
$$

where $\operatorname{area}(\cdot)$ is area of membership function.

Definition 2.2. We define the maximum grade membership function of rejection or acceptance degree by agreement index for real-valued function $\Re_{\delta}$ by $\delta$-level as

$$
\begin{aligned}
& \Re_{\delta}(0)=\sup _{\theta}\left\{\frac{\text { area }\left(\mathrm{m}_{\mathrm{T}_{\delta}}(\theta) \cap \mathrm{m}_{\mathrm{C}_{\delta}}(\theta)\right)}{\text { area } \mathrm{m}_{\mathrm{C}_{\delta}}(\theta)}\right\}, \\
& \Re_{\delta}(1)=1-\Re_{\delta}(0)
\end{aligned}
$$

by $\delta$-level for the fuzzy hypothesis testing.

In agreement index, we have the area by $\delta$-level as;

$$
\begin{aligned}
& \operatorname{area}\left(m_{C}(x) \cap m_{T}(x)\right)= \\
& \quad \int_{\delta_{0}}^{\delta_{1}}\left(C_{r}^{-1}(\delta)-T_{l}^{-1}(\delta)\right) d \delta
\end{aligned}
$$

and

$$
\text { area } m_{C_{\delta}}(\theta)=\int_{\delta_{0}}^{1}\left(C_{r}^{-1}(\delta)-C_{l}^{-1}(\delta)\right) d \delta
$$


where $C_{r}, C_{l}$ are right and left side line of $m_{C}(x)$, $X_{l}$ is left side line of $m_{T}(x)$ and $\delta_{0}$ is reliable degree and $\delta_{1}$ is meeting point of $m_{C}(x)$ and $m_{T}(x)$.

\section{Fuzzy significance probability}

A fuzzy test of the fuzzy null hypothesis is a course of separation of a fuzzy set if values of a fuzzy random variable $X$ for which $H_{f 0}$ is would be rejected. The fuzzy random variable whose value serves to determinable the action is called the fuzzy test statistic, and the fuzzy set of its values for which $H_{f 0}$ is to be rejected is called the fuzzy rejection region of the test. A fuzzy test of specifiable degree by a fuzzy test statistic and the fuzzy rejection region is denoted by (2.2) and (2.3).

For the fuzzy hypothesis, we consider

$$
H_{f 0}: \theta \simeq \psi \text { or } H_{f 0}: \theta<\psi, \theta \in \Theta \text {. }
$$

If we have $\mathrm{D}$ test statistics $T$ then the significance probability is given by $P(T>x)$ or $P(T<x)$.

If the fuzzy significance probability is $P(T<x)>\alpha$ then we reject the hypothesis where $\alpha$ is fuzzy significance level. Considering the unknown state of nature and the possible results from applying a fuzzy test, one of the following situations will arise:

\begin{tabular}{|c|c|c|}
\hline & \multicolumn{2}{|c|}{ UNKNOWN TRUE STATE OF } \\
\hline $\begin{array}{c}\text { TEST } \\
\text { concludes }\end{array}$ & $\begin{array}{c}H_{f 0} \text { true } \\
\theta<\psi\end{array}$ & $\begin{array}{c}H_{f 0} \text { false } \\
\theta>\psi\end{array}$ \\
\hline $\begin{array}{c}\text { Do not reject } \\
H_{f 0}\end{array}$ & $\begin{array}{c}\text { Correct } \\
\text { degree }\end{array}$ & $\begin{array}{c}\text { Wrong degree } \\
\text { (type } \amalg \text { error) }\end{array}$ \\
\hline Reject $H_{f 0}$ & $\begin{array}{c}\text { Wrong degree } \\
\text { (type I error) }\end{array}$ & $\begin{array}{c}\text { Correct } \\
\text { degree }\end{array}$ \\
\hline
\end{tabular}

Fuzzy Type I error : rejection degree of $H_{f 0}$ when
$H_{0}$ is true.

Fuzzy Type $\Pi$ error : failure degree to reject $H_{f 0}$ when $H_{1}$ is true.

The probabilities of the two types of error $\alpha=P[$ type $\mathrm{I}$ error $]=P\left[\right.$ rejection degree of $H_{f 0}$ when $H_{f 0}$ is true],

$\beta=P[$ type $\Pi$ error $]=P\left[\right.$ not rejecting degree $H_{f 0}$ when $H_{f 1}$ is true].

The fuzzy probability $\alpha$ depends on the particular value of the parameter in the range covered by $H_{f 0}$, whereas $\beta$ depends on the value over the range covered by $H_{f 1}$ and $\gamma(\theta)=P$ [the fuzzy test rejects $H_{f 0}$ when the true value of the parameter is $\left.\theta\right]$.

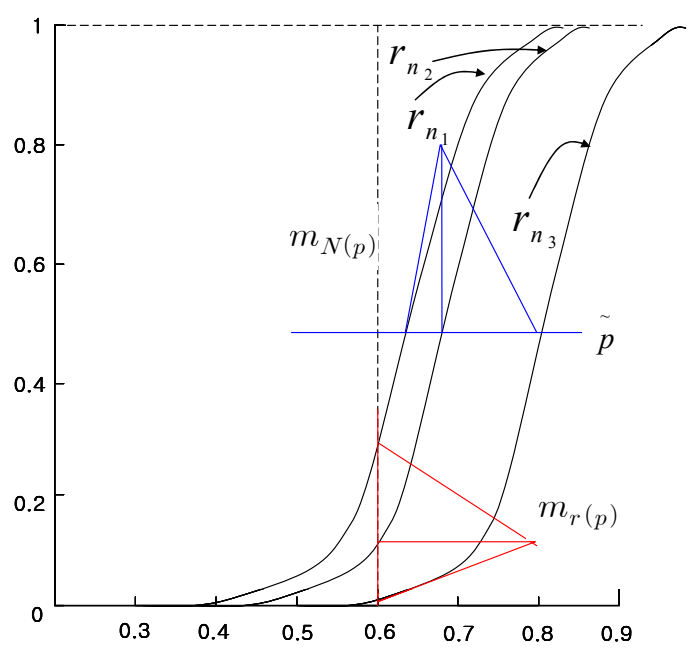

Fig. 1 A power curves

Under $H_{f 0}, \theta$ is restricted to the range $\theta<\psi$, which is to the left of the middle vertical membership function in Fig. 1 In this part of the graph, the rejection probability $\gamma(\theta)$ is, by definition, the same as the type I error fuzzy probability $\alpha(\theta)$.

Under $H_{1}$, the range of $\theta$ is $\theta>\psi$, which is to the right of the middle vertical membership function. In this range, $1-\gamma(\theta)=P\left[\right.$ retain $\left.H_{f 0}\right]=P[$ type $\square$ 
error $]=\beta(\theta)$. Thus the graph of the fuzzy rejection probability curve $\gamma(\theta)$ of a test provides a complete picture of the performance of the test for all possible contingencies with regard to the true degree state of fuzzy nature.

\section{Drawing conclusions}

For drawing a conclusion, a researchers have fuzzy number samples by fictitious data for modulus of elasticity(Gpa) by Young's modulus of collected 10 amount of work for iron as;

[174, 176], [188, 192], [213, 217], [196, 200],

$[183,185],[205,209],[207,213],[191,195]$,

[194, 198], [179, 181].

The data indicate a cause for concern that modulus of elasticity(Gpa) is about 202. Thus, the null hypothesis should be

$$
H_{f 0}: \mu \simeq \widetilde{202} .
$$

Since the counts are spread over a wide range, an approximation by a continuous distribution is not unrealistic for inference about the fuzzy mean.

Assuming that the measurements consistence a fuzzy sample from a normal population and that the level of significance $\alpha=0.1$. We employ the $t$-test

$$
\tilde{t}=\frac{\tilde{\bar{X}}-\widetilde{202} \mid}{\tilde{s} / \sqrt{10}}
$$

with rejection region $t \leqq-t_{0.1 / 2}$ or $t \geqq t_{0.1 / 2}$ From the $t$-table, we determine that $t_{0.05}=1.833$ with d.f. $=9$.

Computations from the sample data yields

$$
\widetilde{\bar{X}}=[193.0+1.8 \delta, 196.6-1.8 \delta],
$$

$$
\begin{aligned}
\widetilde{s^{2}}=[106.311 & +51.511 \delta+14.8 \delta^{2}, \\
& \left.268.533-110.711 \delta+14.8 \delta^{2}\right] .
\end{aligned}
$$

If we have $\delta=1$,

$$
\begin{aligned}
\tilde{t}=\frac{|\widetilde{\bar{X}}-202|}{\tilde{s} / \sqrt{10}} & =[1.733,1.733] \\
& <t(0.05 ; 9)=1.833 .
\end{aligned}
$$

By (4.2), we accept the fuzzy alternative hypothesis $H_{f 0}$. If $\delta=0.0$ then we have

$$
\tilde{t}=\frac{|\widetilde{\bar{X}}-[201+\delta, 203-\delta]|}{\tilde{s} / \sqrt{10}}=[0.849,3.067]
$$

and $t(0.05 ; 9)=1.833$.

From (2.2) and (2.3), we have

$$
\begin{aligned}
& \int_{0}^{0.927}(-1.333 \delta+3.067-1.833) d \delta / \\
& \int_{0}^{1}((-1.333 \delta+3.067)-(0.884 \delta+0.849)) d \delta \\
&= 0.516
\end{aligned}
$$

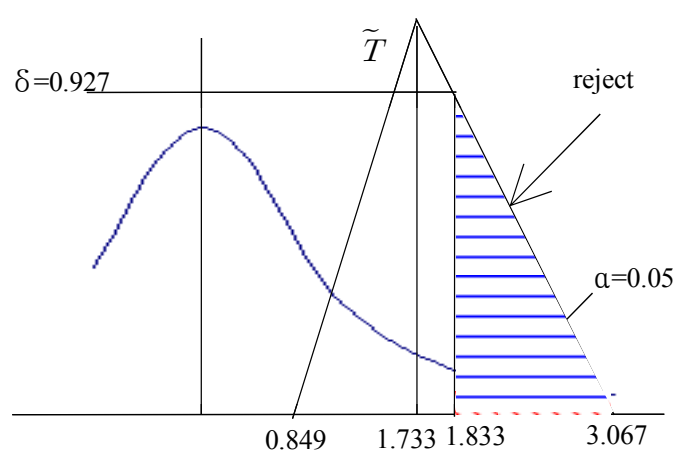

Fig. 2 Rejection degree by agreement index

Thus, we accept the fuzzy alternative hypothesis $H_{f 0}$ degree of $\Re_{\gamma}(0)=0.516$ by [Definition 3.1] as [Fig. 2], and we can show that the membership function of rejection degree by significance 
probability to [Fig. 3].

Another hand, we can show the rejection degree of the fuzzy hypothesis $H_{f 0}$ by significance probability as $\Re_{\gamma}(0)=0.163$ by [Definition 3.1] and [Fig. 4].

We can compare the properties of the fuzzy test statistics with fuzzy number samples, the fuzzy test by significance probability is excellent test for statistical hypothesis rather than crisp $t$-test.

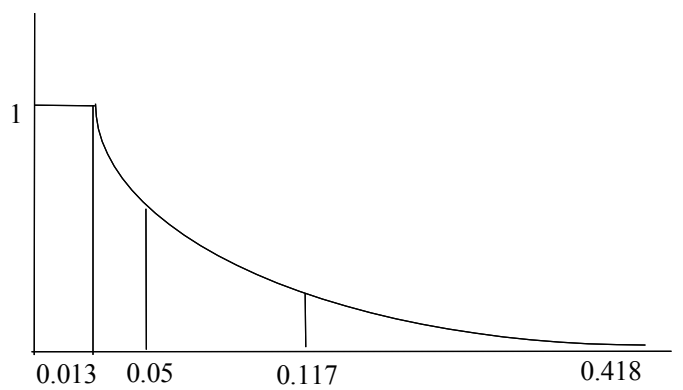

Fig. 3 Rejection degree by significance probability

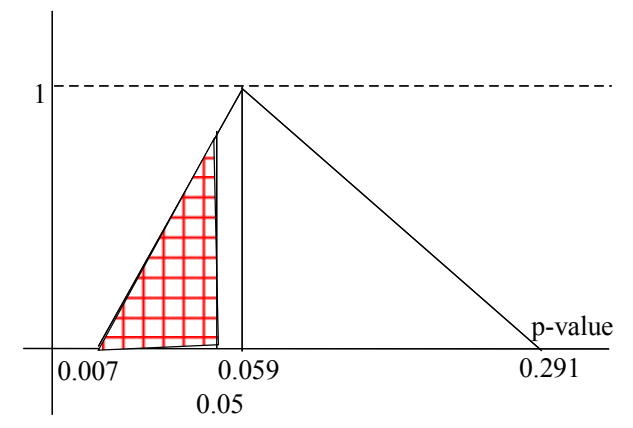

Fig. 4 Degree of significance probability

\section{References}

1. P. X. Gizegorzewski, 2000, "Testing Hypotheses with vague data", Fuzzy Sets and Systems. 112, pp. 501-510.

2. M. K. Kang, G. T. Choi and C. E. Lee, 2000, "On Statistical Test for Fuzzy Hypotheses with Fuzzy Data", Proceeding of Korea Fuzzy Logic and Intelligent System Society Fall Conference,
Vol. 10, Num. 2.

3. M. K. Kang, G. T. Choi and S. I .Han, 2000, “A Bayesian Fuzzy Hypotheses testing with Loss Function”, Proceeding of Korea Fuzzy Logic and Intelligent Systems Society Fall Conference, Vol 13, Num. 2.

4. M. K. Kang, Lee, C. E. and Han, S. I. , 2003, "Fuzzy Hypotheses Testing for Hybrid Numbers by Agreement Index", Far East Journal of Theoretical Statistics., 10(1).

5. S. F. Schnatter, S., 1992, "On Statistical inference for Fuzzy Data whit Application to Des Crispy Statistics”, Fuzzy Sets and Systems, 50, pp 143-165. 